\title{
ac response of Hg-based superconductors: Surface barrier and bulk pinning contributions
}

\author{
L. Fàbrega, A. Sin,* A. Calleja, and J. Fontcuberta \\ Institut de Ciència de Materials de Barcelona (CSIC), Campus de la UAB, 08193 Bellaterra, Spain
}

(Received 29 November 1999)

\begin{abstract}
A complete study of the ac response of $\mathrm{Hg}$-based superconductors with 1, 2, and $3 \mathrm{Cu}-\mathrm{O}$ planes is presented. Under a dc field, the ac susceptibility $\chi_{\mathrm{ac}}(T)$ displays a double transition, which is argued to be of intrinsic nature. The systematic analysis of the evolution of $\chi_{\mathrm{ac}}$ (fundamental and higher harmonics) as a function of the experimental parameters, and the comparison with resistance and dc magnetization measurements, suggest that the higher temperature transition is ascribed to magnetic irreversibility due to surface barriers; the lower temperature transition is associated to bulk pinning.
\end{abstract}

\section{INTRODUCTION}

Early after the discovery of high- $T_{c}$ superconducting (HTSC) cuprates, it became clear that the characterization of the dissipation phenomena taking place in the mixed state of these materials was a fundamental issue. The high critical temperature $T_{c}$, extreme type-II character, and large anisotropy of these superconductors leads to unusually important effects of thermal fluctuations, which reflect themselves in the existence of a variety of new vortex phases, and thermally activated flux motion even at low driving currents. From the point of view of practical applications, this landscape leads to the existence of a characteristic line $H_{\text {irr }}(T)$ far from the upper critical field, the so-called irreversibility line (IL), above which the superconductor can carry no current without energy dissipation. The determination of this line and its origin is hence a key point to evaluate the possible technological uses of each HTSC material.

ac susceptibility is a powerful tool to determine the IL of superconductors and its origin. The peak in the out-of-phase component of the fundamental ac susceptibility $\chi_{1}$ and the appearance of a contribution to the third harmonic component $\chi_{3}$-ascribed, respectively, to the energy losses and magnetic hysteresis - have been used to define $H_{\text {irr }}(T){ }^{1,2}$ ac losses in the mixed state of type-II superconductors are due to vortex motion, which may be reversible or irreversible. In the former case, the motion is diffusionlike, and the ac response is linear. In the latter case, nonlinear effects and higher harmonics should become apparent, and two frameworks have been considered: bulk pinning (within the critical-state model) and surface or geometrical barriers. Additionally, thermally activated flux motion-very common in HTSC_-produces a frequency-dependent ac response, which may be used to extract the effective activation energies for these processes.

$\mathrm{Hg}$-based HTSC are extremely interesting due to their high $T_{c}$ (134 $\mathrm{K}$ for the $\mathrm{HgBa}_{2} \mathrm{Ca}_{2} \mathrm{Cu}_{3} \mathrm{O}_{8}$ compound). In spite of it, detailed studies of dissipation in these materials are scarce; this fact might be partially due to the difficulties to obtain high-quality samples of these phases. The IL has been determined mainly from dc magnetization measurements; it is found to lie quite low-for instance, that of Hg-1223 lies below that of the isostructural Tl-1223 phase. ${ }^{3,4}$ The width of the hysteresis cycles decreases very fast when increasing field and temperature, and they become highly asymmetric at rather low temperatures and fields $(T \sim 10 \mathrm{~K}$ for the $\mathrm{Hg}-1201$ phase $^{5,6}$ and $T \sim 40 \mathrm{~K}$ for the (Hg-Re)-1223 phase $^{7}$ ); this fact is interpreted as due to the dominant contribution of surface barriers over bulk pinning. ${ }^{5-7}$ The remarkable presence of surface barriers in a broad range of fields and temperatures, for all the members of this family of HTSC, is striking; it is not well understood nowadays, and has been attributed to a low density of defects, both at surface and in the bulk. ${ }^{5}$

In this paper we perform a detailed study of the energy dissipation associated with flux motion, on high-quality superconducting ceramics and powders of $\mathrm{Hg}$-based superconductors, by using mainly ac susceptibility $\chi_{\text {ac }}$ and also transport and dc magnetization measurements. Under a dc field, all the studied samples display-aside from the intergrain transition - the systematic presence of a double ac transition, revealed by two diamagnetic steps in the in-phase component of the fundamental ac susceptibility $\chi_{1}^{\prime}$ and two dissipation peaks in the out-of-phase component, $\chi_{1}^{\prime \prime}$. From the study of the evolution of $\chi_{\mathrm{ac}}$ (fundamental and higher harmonics) with dc field, ac field amplitude and frequency, and its comparison with resistance $R$ and magnetization $M$ data as a function of temperature, it is concluded that the two transitions may be associated, respectively, with bulk pinning and surface barriers. The study of the ac susceptibility of these compounds brings therefore a unique opportunity to learn about the ac response in the case that magnetic irreversibility is dominated by surface barriers and a weak bulk pinning is present, a rather unusual case within HTSC materials.

\section{EXPERIMENTAL}

The measured samples had compositions $\mathrm{HgBa}_{2} \mathrm{CuO}_{4}$ (HBCO), $\quad \mathrm{Hg}_{0.82} \mathrm{Re}_{0.18} \mathrm{Sr}_{2} \mathrm{CaCu}_{2} \mathrm{O}_{6} \quad$ (HRSCCO), and $\mathrm{Hg}_{0.82} \mathrm{Re}_{0.18} \mathrm{Ba}_{2} \mathrm{Ca}_{2} \mathrm{Cu}_{3} \mathrm{O}_{8}$ (HRBCCO); they were synthesized by the sealed quartz tube technique, as described elsewhere. ${ }^{8}$ The experimental features reported in this paper have been observed in all these samples, although here we will concentrate on the HRBCCO samples, for which the analyses performed are more complete.

Transport and some ac susceptibility measurements were performed on ceramic bars with typical dimensions $2 \times 2$ $\times 6 \mathrm{~mm}^{2}$. ac susceptibility $(n=1,2$, and 3 harmonics) was 


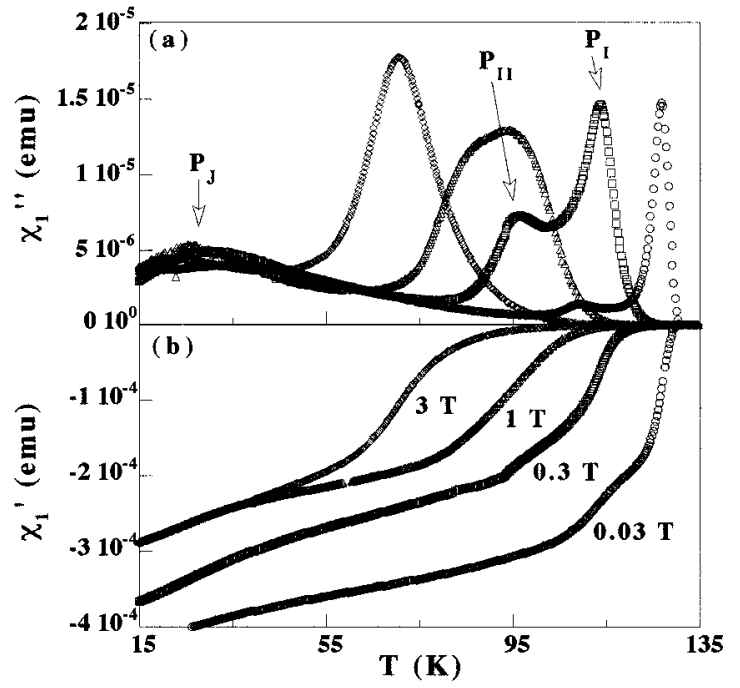

FIG. 1. Temperature dependence of the in- and out-of-phase components $\chi_{1}^{\prime}$ and $\chi_{1}^{\prime \prime}$ of the fundamental ac susceptibility $\chi_{1}$ of a HRBCCO powder (weakly ground), recorded with $h_{\mathrm{ac}}=1 \mathrm{Oe}$ and $f=1111 \mathrm{~Hz}$, at several applied dc fields $H$. The three peaks $P_{\mathrm{J}}$, $P_{\mathrm{II}}$, and $P_{\mathrm{I}}$ discussed in the text are indicated for the data recorded at $0.3 \mathrm{~T}$.

measured in a Commercial system [Physical Property Measurement System (PPMS), from Quantum Design], with applied dc magnetic fields up to $9 \mathrm{~T}$ parallel to the ac field. Both fields were applied along the long dimension of the sample bars. The ac field amplitude $h_{\text {ac }}$ and frequency $f$ were changed between 0.3 and $12.7 \mathrm{Oe}$, and 33 and $8789 \mathrm{~Hz}$, respectively. The experiments were performed in fieldcooling (FC) conditions, in order to have a homogeneous vortex distribution inside the sample, and with $h_{\mathrm{ac}} \ll H$. Some measurements were also performed on powder samples, in order to reduce the contribution of intergrain effects at low temperatures.

Transport measurements were carried out on ceramic bars in the PPMS equipment. Resistance was measured by the conventional four-probe technique, using driving currents between $10 \mu \mathrm{A}$ and $5 \mathrm{~mA}$. The noise threshold was 0.1 $\mu \mathrm{V} / \mathrm{cm}$; this value was used as the criterion to define the resistance onset.

Additionally, temperature-dependent dc magnetization (ZFC and FC curves) was also measured in a commercial (Quantum Design) superconducting quantum interference device.

All the studied samples display a single and sharp transition in $\chi_{\mathrm{ac}}(T)$ and $R(T)$ when $H=0$. dc magnetization at low fields displays also a single, sharp diamagnetic step. ${ }^{9}$ These transitions allow to define the critical temperatures of these phases, from the onsets of diamagnetism or resistance drop; they are 97, 107, and $133 \mathrm{~K}$ for the HBCO, HRSCCO, and HRBCCO compounds, respectively.

\section{RESULTS}

\section{A. Fundamental ac susceptibility}

Figures 1(a) and 1(b) display the temperature dependence of the in- and out-of-phase components of the fundamental ac susceptibility, $\chi_{1}^{\prime}$ and $\chi_{1}^{\prime \prime}$, for a HRBCCO powder (weakly ground in an agathe mortar), at several external dc fields $H$. When lowering $T$, a first diamagnetic transition takes place, accompanied by a peak $P_{1}$ in $\chi_{1}^{\prime \prime}$. As $H$ increases, both the peak and the diamagnetic step shift to lower temperatures; the height of the peak is not sensibly affected by $H$. At finite dc fields, a second diamagnetic steptogether with another peak $P_{\mathrm{II}}$ in $\chi_{1}^{\prime \prime}$-occurs at temperatures somehow lower. This peak experiences a considerable development with dc field, and shifts to lower temperatures. Neither $P_{\mathrm{I}}$ nor $P_{\mathrm{II}}$ display a significant broadening for increasing $H$. At high fields $\left(\mu_{0} H>1 \mathrm{~T}\right)$, however, both peaks $P_{\mathrm{I}}$ and $P_{\mathrm{II}}$ approach and appear to merge in a single one (see Fig. 4 and discussion below). Finally, a third transition towards higher ac screening, with its peak $P_{J}$, appears at low temperatures. This peak is very sensitive to magnetic fields: it shifts to lower temperatures, broadens and is smeared out for rather low values of $H$. Besides, it is considerably weaker in powder samples than in ceramics, and disappears in plastically deformed samples, for which many cracks and microcracks are induced; ${ }^{10}$ therefore, it may be ascribed to intergrain coupling, still present in the powder, and will not be further discussed in this paper.

The interpretation of the other two peaks $P_{\mathrm{I}}$ and $P_{\mathrm{II}}$ is less clear. While, at first, $P_{\mathrm{I}}$ might be associated to the intrinsic ac response of the superconducting grains, the existence of the extra peak $P_{\mathrm{II}}$ is quite puzzling, and might even make not so evident the interpretation of $P_{\mathrm{I}} . P_{\mathrm{II}}$ has been observed in all the powder and ceramic samples we have measured; its behavior with the different experimental parameters-which will be fully described below- is reproducible and systematic, although its height relative to $P_{\mathrm{I}}$ may change for different samples and compounds. This is shown in Fig. 2, which displays the $\chi_{1}(T)$ data at $\mu_{o} H=0.1 \mathrm{~T}$ for three samples corresponding to the three measured phases. All these samples are single phased, and contain only minor traces of second phases (as typically found in $\mathrm{Hg}$-based superconducting ceramics, due to their unstability and difficult synthesis), so that the peak may hardly be due to some other lower $T_{c}$ phase; this deduction is reinforced by the fact that the relative height between the $P_{\mathrm{I}}$ and $P_{\mathrm{II}}$ peaks depends on the external field (see Figs. 1 and 7). In fact, the rather robust behavior of the peak with external dc field-as well as with ac field amplitude, as we shall see-denies its ascription to some Josephson coupling due, for instance, to low-angle grain boundaries. This conclusion is further confirmed by the fact that extra grindings, which result in a marked decrease of the average grain size, do not alter the relative height of the $P_{\mathrm{I}}$ and $P_{\mathrm{II}}$ peaks, nor their position. This is clearly appreciated in Fig. 3, where the $\chi_{1}^{\prime \prime}(T)$ data at $\mu_{o} H=0.1 \mathrm{~T}$ is shown for a HRBCCO powder ground once and twice: in spite of a considerable decrease of the superconducting volume fraction-which has been associated with the fact that the average grain size becomes comparable to the penetration depth - the dissipation peaks remain basically unchanged. Even more, room-temperature plastic deformation, besides producing many cracks and microcracks-and thereby reducing also the grain size and destroying intergrain coupling ${ }^{10}$-increases the height of $P_{\mathrm{II}}$ relative to $P_{\mathrm{I}} \cdot{ }^{11}$

In order to determine the origin of these two peaks, we have compared the ac data with resistance and dc magnetization measurements, and measured the evolution of the fun- 


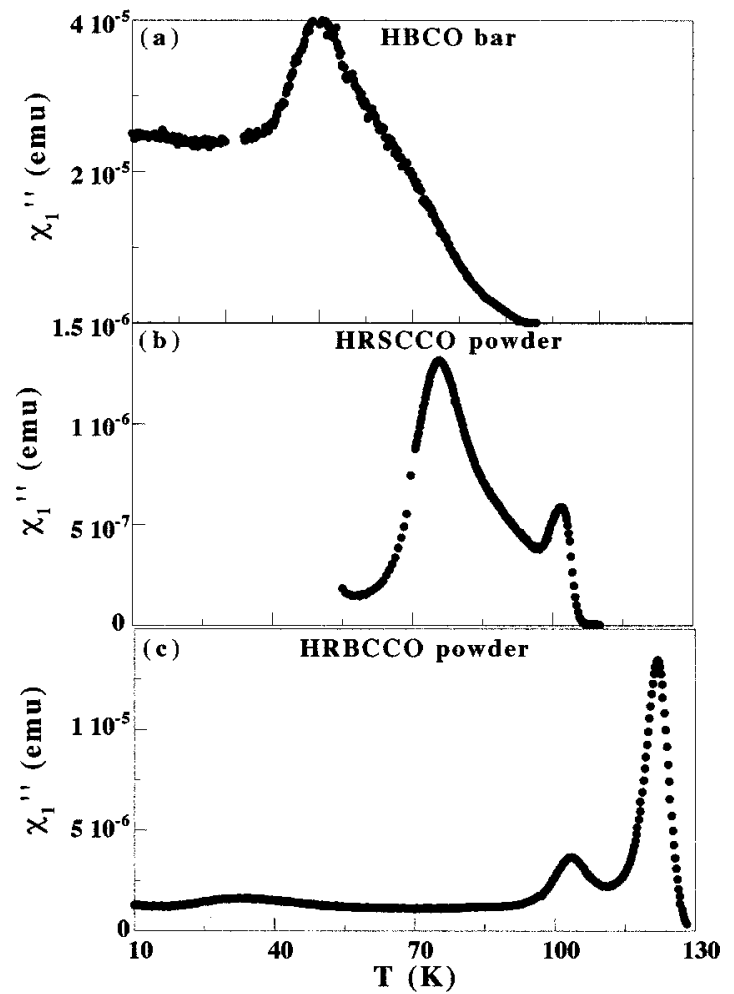

FIG. 2. $\chi_{1}^{\prime \prime}(T)$ recorded at $\mu_{0} H=0.1 \mathrm{~T}$ and $h_{\mathrm{ac}}=1$ Oe for three samples of different phases: $\mathrm{HBCO}\left(T_{c}=97 \mathrm{~K}\right), \operatorname{HRSCCO}\left(T_{c}\right.$ $=107 \mathrm{~K})$ and $\operatorname{HRBCCO}\left(T_{c}=133 \mathrm{~K}\right)$.

damental and higher harmonics $(n=2,3)$ with the external dc field, and the ac field amplitude and frequency. The obtained results are described in the following subsections. In the next section a discussion and interpretation of the experimental data within the available theoretical frameworks are given.

\section{B. Comparison to transport and dc magnetization}

Figure 4 displays the temperature dependence of $\chi_{1}^{\prime}$ and $\chi_{1}^{\prime \prime}$ of a HRBCCO powder, and the resistance $R$ of a bar of the same sample, at several selected dc fields. Resistance was measured at several currents between $10 \mu \mathrm{A}$ and $5 \mathrm{~mA}$, and no differences in the $R(T)$ curves were detected within this current range, corresponding to low driving current densities $\left(J \leqslant 0.125 \mathrm{~A} / \mathrm{cm}^{2}\right)$. The $R(T)$ curves look very much the same as those reported for Re-free Hg-1223 ceramics; ${ }^{4}$ the onset of resistance drop is nearly field independent, while the

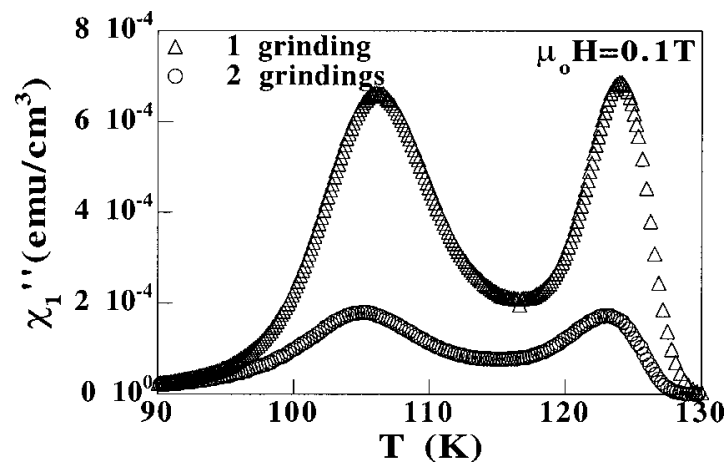

FIG. 3. $\chi_{1}^{\prime \prime}(T)$ at $\mu_{0} H=0.1 \mathrm{~T}$ and $h_{\mathrm{ac}}=1.27$ Oe for a 1223 powder ground once and twice.

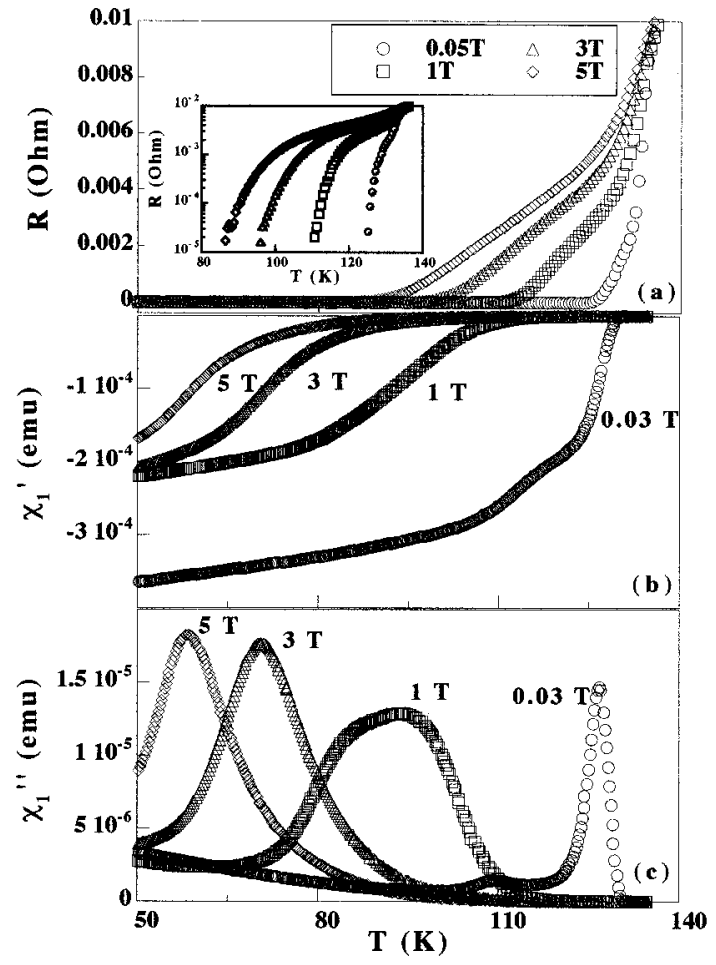

FIG. 4. Temperature dependence of $R(\mathrm{a}), \chi_{1}^{\prime}(\mathrm{b})$, and $\chi_{1}^{\prime \prime}$ (c) for a HRBCCO sample, at several applied dc fields $H$. Resistance data belong to a ceramic bar, and ac data were measured (with $h_{\mathrm{ac}}$ $=1.27 \mathrm{Oe}$ ) in a weakly ground powder of the same sample, in order to minimize the intergrain contribution. The inset in (a) is a blowup of the onset of resistance, in logarithmic scale.

temperature at which $R$ becomes zero decreases as $H$ increases; this results in a transition broadening with the dc field. Although the critical temperatures of our sample and that of Ref. 4 are the same, the lower part of the transition occurs at higher temperatures in the HRBCCO compound. This shift is likely related to the partial Re substitution for $\mathrm{Hg}$, which has been reported to enhance magnetic irreversibility in these HTSC. ${ }^{7,12}$

Comparison of the ac and transport data in Fig. 4 reveal that the onsets of diamagnetism and ac losses are nearly coincident with the disappearance of resistance in the sample. This feature, which would be canonical for a single-crystal sample with a unique ac transition, is quite amazing in this case: it means that there is a broad temperature range where the measured resistance throughout the sample is zero, although the ac losses appear and attain maximum values at much lower temperatures and fields, as Fig. 5 more clearly reveals. Particularly, this implies that the Josephson junctions between grains display a broad spectrum of strengths, and that some coupling persists up to quite high fields and temperatures, providing a percolative path for currents throughout the sample. A similar conclusion was driven for the same compounds, from ac measurements, in Ref. 5.

Figure 6 displays the characteristic lines of a HRBCCO sample, corresponding to the onset of finite resistance, to the $P_{\mathrm{I}}$ and $P_{\mathrm{II}}$ peaks of $\chi_{1}^{\prime \prime}$, and to the onset of dc magnetic irreversibility, as obtained from temperature-dependent magnetization data [zero-field cooling (ZFC) and field-cooling (FC) processes]. The three criteria are commonly used to define the IL of HTSC. As observed in Figs. 4 and 5, it may 


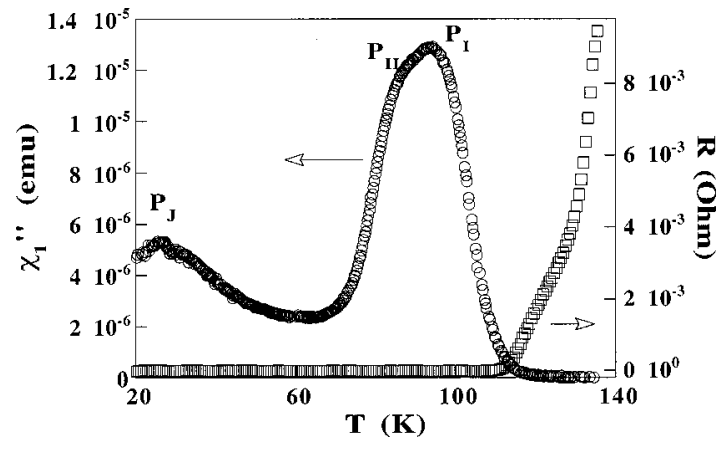

FIG. 5. Comparison of the $R(T)$ and $\chi_{1}^{\prime \prime}(T)$ curves obtained at 1 $\mathrm{T}$ for a HRBCCO sample, measured, respectively, at $1 \mathrm{~mA}$ and 1 Oe. It may be clearly seen that the measured resistance remains zero well above the peak associated to intergrain currents $P_{\mathrm{I}}$, and even $P_{\mathrm{II}}$. The same result is obtained at any field $H$.

be appreciated that at high temperatures and low fields the disappearance of resistance is nearly coincident with the first ac dissipation peak $P_{\mathrm{I}}$. As the field increases and the transition broadens, these two lines separate. One can also observe that $P_{\mathrm{I}}$ coincides with the onset of reversible dc magnetization in a ZFC-FC measurement. Finally, comparison of the lines obtained from $P_{\mathrm{I}}$ and $P_{\mathrm{II}}$ clearly illustrates the behavior of both peaks, which tend to merge for increasing fields, as already discussed. In fact, the points corresponding to 3 and $5 \mathrm{~T}$ might correspond to either $P_{\mathrm{I}}$ or $P_{\mathrm{II}}$. The evolution with the dc field of the features associated to both peaks does not allow to clearly discern whether the single peak observed at these high fields is $P_{\mathrm{I}}, P_{\mathrm{II}}$ or both.

\section{Evolution of the fundamental and higher harmonics with $H, h_{\text {ac }}$ and $f$}

Figures 7, 8, and 9 display the temperature dependence of $\chi_{1}^{\prime \prime}$ and of the moduli of the second and third harmonics, $\chi_{2}$ and $\chi_{3}$, for different values of $H, h_{\mathrm{ac}}$, and $f$, respectively. Analysis of these figures indicates that the dissipation peaks $P_{\mathrm{I}}$ and $P_{\mathrm{II}}$ in $\chi_{1}^{\prime \prime}$ have their corresponding features in $\chi_{2}$ and $\chi_{3}$ : when $P_{\mathrm{I}}$ occurs, peaks in $\chi_{3}$ and $\chi_{2}$ become apparent. Remarkably, the peak $P_{\mathrm{II}}$ appears only accompanied by a peak in $\chi_{2}$ : at low $\mathrm{dc}$ and ac fields no features in $\chi_{3}$ are

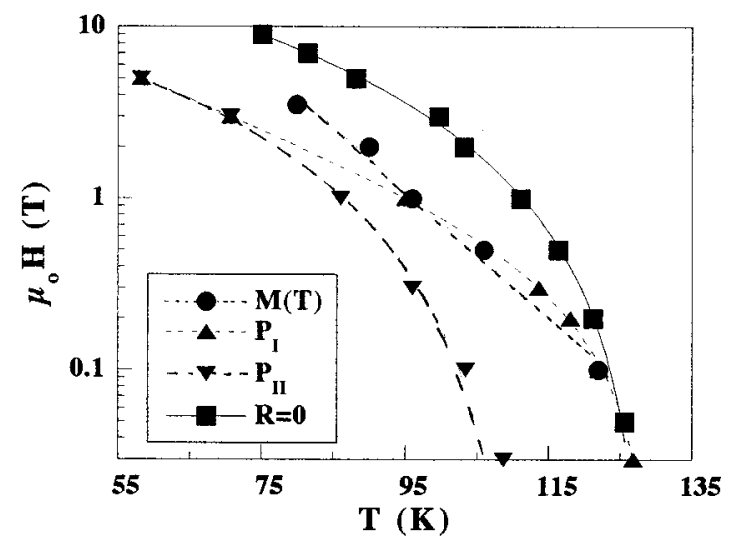

FIG. 6. Characteristic, irreversibility, lines for a HRBCCO sample, obtained from (i) the onset of finite resistance, (ii) the onset of reversible dc magnetization, and (iii) the peaks $P_{\mathrm{I}}$ and $P_{\mathrm{II}}$ of $\chi_{1}^{\prime \prime}$. The lines through the data points are guides for the eyes.
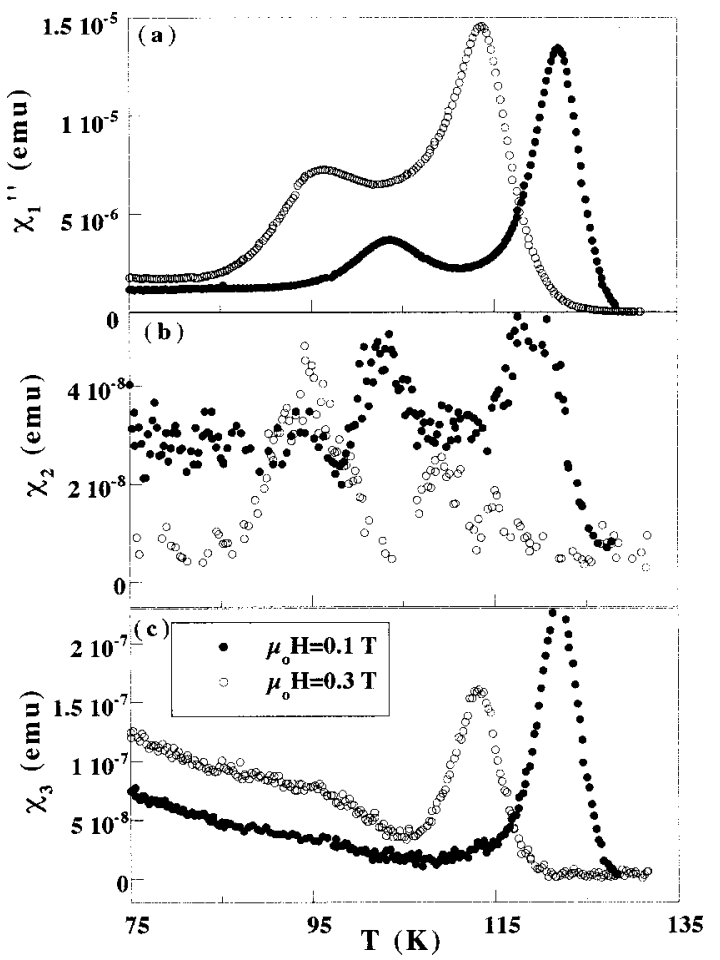

FIG. 7. Temperature dependence of $\chi_{1}^{\prime \prime}$ (a), and the moduli of the higher harmonics $\chi_{2}(\mathrm{~b})$, and $\chi_{3}(\mathrm{c})$, for two applied dc fields (HRBCCO powder, $h_{\text {ac }}=1$ Oe, $f=1111 \mathrm{~Hz}$ ).

observed at the temperature at which $P_{\mathrm{II}}$ occurs. Nevertheless, a weak contribution to $\chi_{3}$ becomes visible at this temperature, at high enough dc and ac fields, as it may be appreciated by the weak shoulders at $\mu_{0} H=1 \mathrm{~T}$ in Fig. 7(c) and at $h_{\mathrm{ac}}=10$ Oe in Fig. 8(c) (see below).

Figure 7 reveals that as the dc field is increased, the features in $\chi_{2}$ and $\chi_{3}$ associated with $P_{\mathrm{I}}$ weaken; those ascribed to $P_{\mathrm{II}}$ either are rather insensitive to $H\left(\chi_{2}\right)$ or increase $\left(\chi_{3}\right)$.

When the ac field amplitude increases (Fig. 8), all the above-mentioned features in the three analyzed harmonics of $\chi_{\mathrm{ac}}$ become more pronounced, indicating that the ac response is highly nonlinear both at $P_{\mathrm{I}}$ and $P_{\mathrm{II}}$. Especially remarkable is the development of the peak in $\chi_{2}$ associated with $P_{\mathrm{II}}$; also, the shoulder on the left of the peak in $\chi_{3}$ seems to indicate the appearance of a contribution to this harmonic, associated with $P_{\mathrm{II}}$, not appreciated at lower $h_{\mathrm{ac}}$ values.

Finally, the evolution of $\chi_{\mathrm{ac}}$ with frequency is shown in Fig. 9. The features in $\chi_{1}^{\prime \prime}, \chi_{2}$, and $\chi_{3}$ associated with $P_{\mathrm{I}}$ shift to higher temperatures for increasing frequencies, as expected when the ac response is due to thermally activated processes. The height of the $P_{\mathrm{I}}$ peak in $\chi_{1}^{\prime \prime}$ remains constant. $P_{\mathrm{II}}$ also shifts to higher temperatures as $f$ is increased; most of this shift might be due to that associated to $P_{\mathrm{I}}$ since both peaks are convoluted. However, the shift of the left-hand side of $P_{\mathrm{II}}$ seems larger than that of $P_{\mathrm{I}}$, indicating that $P_{\mathrm{II}}$ displays some frequency dependence on its own.

Concerning the higher harmonics, the peak in $\chi_{3}$, ascribed to $P_{\mathrm{I}}$, decreases and narrows for increasing frequencies: this narrowing is associated to a shift towards higher temperatures of the left-hand side of the peak, whereas the right-hand side is almost insensitive to frequency. This behavior of $\chi_{3}$ with frequency has been reported for low- $T_{c}$ 


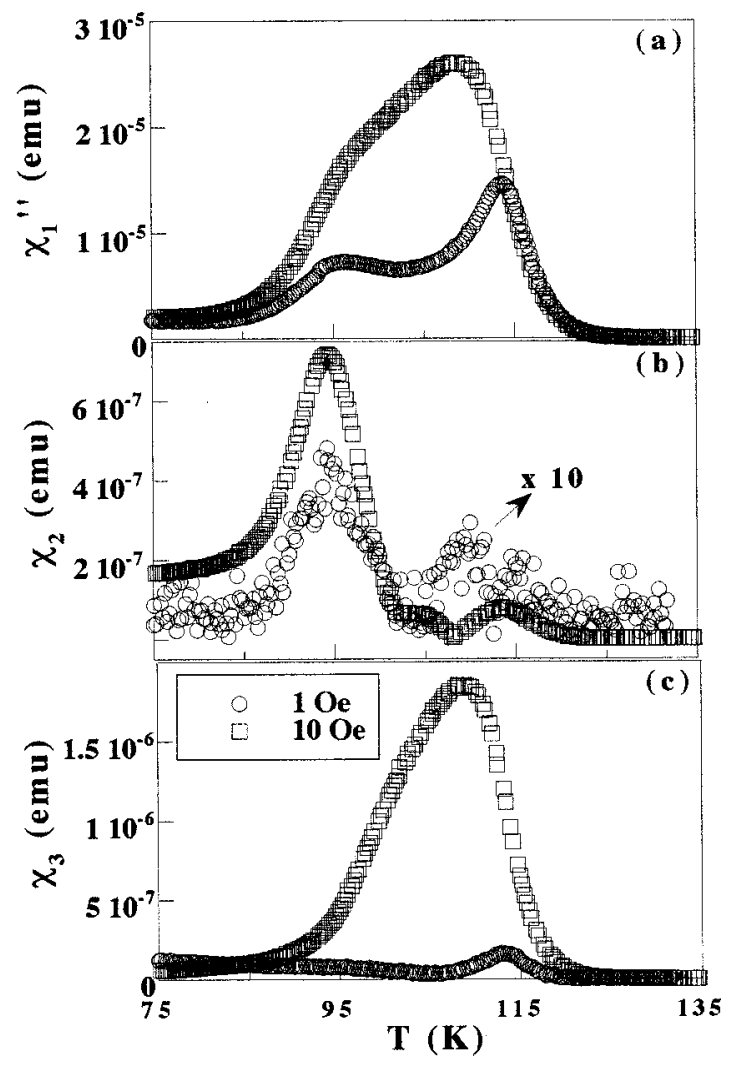

FIG. 8. Temperature dependence of $\chi_{1}^{\prime \prime}$ (a), and the moduli of the higher harmonics $\chi_{2}$ (b), and $\chi_{3}$ (c), for two driving field amplitudes $h_{\mathrm{ac}}\left(\mathrm{HRBCCO}\right.$ powder, $\left.\mu_{0} H=0.3 \mathrm{~T}, f=1111 \mathrm{~Hz}\right)$.

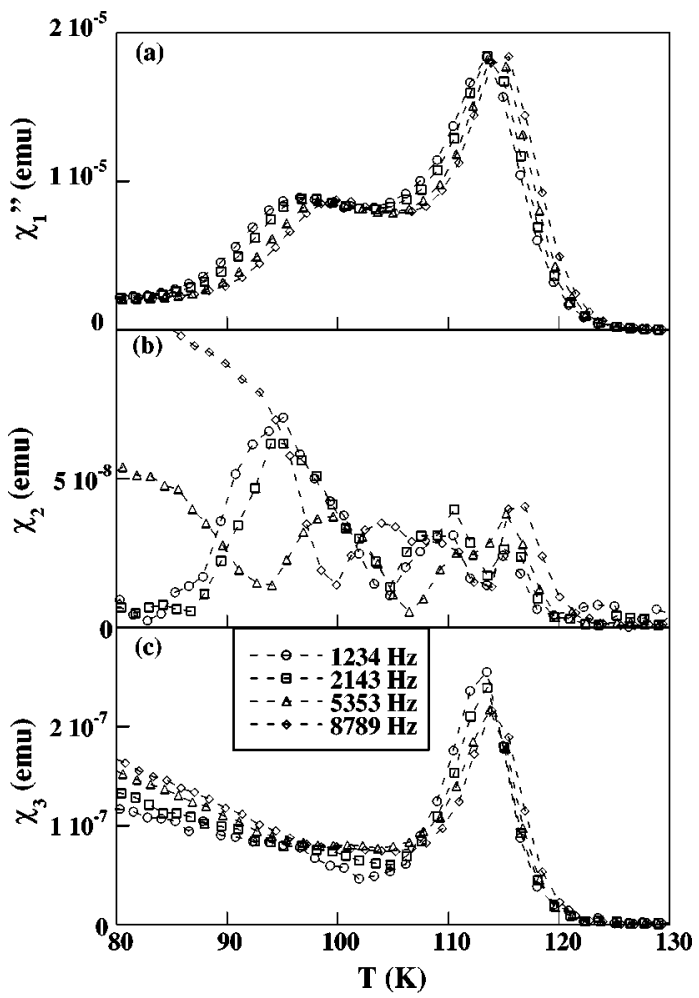

FIG. 9. Temperature dependence of $\chi_{1}^{\prime \prime}$ (a), and the moduli of the higher harmonics $\chi_{2}(\mathrm{~b})$, and $\chi_{3}(\mathrm{c})$, for several frequencies (HRBCCO powder, $h_{\mathrm{ac}}=1.27 \mathrm{Oe}, \mu_{0} H=0.3 \mathrm{~T}$ ). superconductors and Bi-based HTSC. ${ }^{13}$ The evolution of $\chi_{2}$ with frequency is not easy to tell, due to the experimental noise and the background contribution of intergrain coupling, which affects specially the temperature range where the peak associated with $P_{\mathrm{II}}$ is observed; it appears, nevertheless, that the weak peak associated with $P_{\mathrm{I}}$ increases for increasing frequencies; it also shifts to higher temperatures, as the related features in $\chi_{1}^{\prime \prime}$ and $\chi_{3}$ do.

\section{DISCUSSION}

We have shown in the previous section that the ac susceptibility of Hg-based superconductors displays a double transition, reflected by two dissipation peaks $P_{\mathrm{I}}$ and $P_{\mathrm{II}}$. We undertake now the analysis of their observed features with the aim of clarifying their origin.

The finite value of the higher harmonics of the ac response is indicative of the existence of magnetic hysteresis both at $P_{\mathrm{I}}$ and $P_{\mathrm{II}}$. The frequency dependence of these peaks (Fig. 9) reveals that the energy losses in both cases are due to some thermally activated processes, and not to a transition of the vortex system. Due to the same reason, they cannot be associated with shape effects of the plateletlike grains of the powders; this conclusion is further confirmed by the invariance of the position of both peaks when the average grain size is considerably reduced (Fig. 3 and Ref. 10).

Hysteretic magnetic behavior and nonzero higher harmonics are expected in the cases of bulk pinning and surface barriers. The contribution of bulk pinning, within the criticalstate model, ${ }^{13,14}$ is by far the most studied. In this case, when the critical current is independent of the magnetic flux $B$ (Bean model), the hysteresis cycles display symmetric increasing and decreasing field branches, so that even harmonics are predicted to be zero. If the critical current displays a strong dependence on $B, \chi_{2 n}$ might have a finite, low value. On the other hand, it has been argued ${ }^{15}$ that when magnetic irreversibility is due to geometrical or surface barriers a significant contribution to even harmonics should exist, due to the highly asymmetric character of the hysteresis cycles in this case.

In our experimental results, when both $P_{\mathrm{I}}$ and $P_{\mathrm{II}}$ occur the second harmonic $\chi_{2}$ has a finite value. At $P_{\mathrm{I}}, \chi_{2}$ is about one order of magnitude smaller than $\chi_{3}$; this small contribution to $\chi_{2}$ might be accounted for by surface barriers or by bulk pinning with a critical current dependent on the internal flux $B$; but $P_{\mathrm{II}}$ is rather anomalous, since at this position $\chi_{2}$ is always at least twice as large as $\chi_{3}$. The clearly different behavior of both peaks, particularly in what regards their associated contributions to higher harmonics, implies that they are likely to by caused by different dissipation mechanisms. In the following we discuss the possible origins of these two peaks within the available data and theoretical frameworks.

It has been shown that the position of the $P_{\mathrm{I}}$ peak corresponds to the disappearance of irreversibility in the dc magnetization (Fig. 6). Detailed analyses of the dc hysteresis cycles in $\mathrm{Hg}$ - and $(\mathrm{Hg}, \mathrm{Re})$-based superconductors ${ }^{5-7}$ allowed to conclude that at these temperatures the magnetic irreversibility is mainly due to surface barriers. It appears thus pertinent to ascribe the ac response at $P_{\mathrm{I}}$ to the flux penetration over surface barriers. 
The ac susceptibility of a superconductor when surface barriers are active has been outlined in Ref. 15. Vortices enter the sample for applied fields higher than the flux penetration field $H_{p}$; due to the repulsion of the screening currents they form a flux droplet at the center of the sample. In the absence of bulk pinning, this droplet expands and contracts during an ac cycle, since the magnitude of the surface currents - and thus their force on the droplet-changes in time following the driving field $h_{\text {ac }}$. Energy absorption from the ac field will occur at high enough temperatures, when the magnetic irreversibility is small enough to allow the ac field amplitude to bridge between the ascending and descending field branches of the hysteresis cycle. This situation is reached at a point $H_{p o}(T)>H_{p}$, when the vortex droplet reaches the sample borders; for $h_{\mathrm{ac}} \ll H$, this will take place roughly when the dc hysteresis loop closes. Dissipation will grow when further increasing the temperature, and then it will vanish as the surface barriers are suppressed. As a consequence, a peak will appear in $\chi_{1}^{\prime \prime}$, which we identify with $P_{\mathrm{I}}$. Theoretical calculations ${ }^{15}$ have shown that this peak is accompanied by a kink in $\chi_{1}^{\prime}$, which looks very much the same as that displayed by our data at $P_{\mathrm{I}}$ (Fig. 1). Even more, if vortices can overcome the surface barriers by thermal activation, these peaks and kinks should display a frequency dependence, as experimentally observed.

If some bulk pinning exists in the sample, it will obstruct the expansions and contractions of the vortex droplet, and thus further prevent the penetration of the ac field at low temperatures and fields. As $T$ rises, bulk pinning becomes increasingly weak, and vortices will be able to overcome it, with the help of thermal activation. This will result in a loss of ac screening and the appearance of another dissipation peak. In the case of the Hg-based superconductors, with weak bulk pinning and surface barriers dominating the magnetic irreversibility in a wide region of the $H$ - $T$ phase diagram, this has to take place at temperatures and fields below the peak associated to these surface barriers. The lower temperature peak $P_{\mathrm{II}}$ in our data might thus have this origin.

Within this framework, the increasing height of $P_{\mathrm{II}}$ with $H$ is easily understood, since the vortex droplet contains more vortices and becomes larger with $H$, and thus more dissipation appears in the sample. Likewise, the frequency dependence of $P_{\mathrm{II}}$ is explained by the thermally activated overcome of the potential wells associated to bulk pinning, so common in HTSC. Also, the complex dependence of the size of the vortex droplet with the ac and dc fields, together with the existence of a finite critical current strongly depen- dent on $B$, might account for the relatively large contribution to $\chi_{2}$ observed at $P_{\mathrm{II}}$.

Finally, the coincidence of the resistance onset with the higher temperature side of $P_{\mathrm{I}}$ implies that the surface critical current ${ }^{16}$ is quite high, and that there is a broad temperature range for which transport currents flow within the surface of the grains, and this prevents the appearance of finite resistance, in spite of the weak bulk pinning.

\section{CONCLUSIONS}

In conclusion, detailed analyses of the transport and ac susceptibility measurements of $(\mathrm{Hg}, \mathrm{Re})$-based superconductors reveal the existence of an intrinsic double ac response, associated with thermally activated flux motion. A plausible scenario is suggested, in which the higher temperaturehigher field transition is due to magnetic hysteresis associated with surface barriers; the lower temperature transition is ascribed to the ac driven jumps of vortices between different bulk pinning wells, in the flux droplet inside the sample.

Crossovers from bulk to surface pinning had been argued to exist in other HTSC mainly in Bi-based materials, both from the shape of the hysteresis cycles and the changes in magnetic relaxation processes. ${ }^{17}$ No ac experiments clearly revealing both contributions to magnetic irreversibility had been reported so far, to our knowledge. The observation of such distinct ac response in $\mathrm{Hg}$-based superconductors may be due to the pronounced surface barriers effects in these materials, which allow the observation of their contribution even when bulk pinning is not negligible. They offer therefore a unique chance to study flux dynamics in this complex case. And, particularly, ac susceptibility seems a very useful technique to detect them, although theoretical background is lacking.

On the other hand, the proposed framework means that the transport critical current remains finite up to temperatures and fields much higher than those at which bulk pinning becomes vanishingly small (note that resistance appears in HRBCCO ceramics above $77 \mathrm{~K}$ at $9 \mathrm{~T}$ ), due to the surface barriers, even in polycrystalline materials. This fact could be of extreme relevance in view of the development of applications with these compounds.

\section{ACKNOWLEDGMENTS}

Financial support from the Spanish CICYT (Project No. MAT99-0984-CO3) is acknowledged. L.F. wishes to thank the M.E.C. (Spain) for her contract.
*Present address: Laboratoire de Crystallographie-CNRS, 25 Avenue des Martyrs, BP 166, F-38042 Grenoble Cedex 09, France.

${ }^{1}$ T. K. Worthington, W. J. Gallagher, D. L. Kaiser, F. H. Holtzberg, and T. R. Dinger, Physica C 153-155, 32 (1988).

${ }^{2}$ L. A. Angurel, F. Lera, A. Badía, C. Rillo, R. Navarro, J. Bartolomé, J. Melero, J. Flokstra, and R. P. J. Ijsselsteijn, in High $T_{c}$ Superconductor Thin Films, Proceedings of the International Conference On Advanced Materials ICAM-91, edited by L. Correra (Elsevier, Amsterdam, 1991).

${ }^{3}$ U. Welp, G. W. Crabtree, J. L. Wagner, and D. G. Hinks, Physica C 218, 373 (1993).

${ }^{4}$ A. Schilling, O. Jeandupeux, J. D. Guo, and H. R. Ott, Physica C
216, 6 (1993).

${ }^{5}$ Y. R. Sun, J. R. Thompson, J. Schwartz, D. K. Christen, Y. C. Kim, and M. Paranthaman, Phys. Rev. B 51, 581 (1995).

${ }^{6}$ Y. R. Sun, J. R. Thompson, H. R. Kerchner, D. K. Christen, M. Paranthaman, and J. Brynestad, Phys. Rev. B 50, 3330 (1994); J. A. Lewis, V. M. Vinokur, J. Wagner, and D. Hinks, ibid. 52, R3852 (1994).

${ }^{7}$ L. Fàbrega, B. Martínez, J. Fontcuberta, A. Sin, A. Piñol, and X. Obradors, Physica C 296, 29 (1998).

${ }^{8}$ A. Sin, L. Fàbrega, M. T. D. Orlando, A.-G. Cunha, S. Piñol, and E. Baggio-Saitovich, X. Obradors, Physica C 328, 80 (1999).

${ }^{9}$ Inductive measurements on ceramic samples display a second 
diamagnetic step and dissipation peak at lower temperatures, due to intergrain coupling, as it will be shown.

${ }^{10}$ L. Fàbrega, A. Sin, S. Piñol, D. Eyidi, and J. Rabier, Physica C 325, 27 (1999).

${ }^{11}$ L. Fàbrega et al. (unpublished).

${ }^{12}$ K. Kishio, J. Shimoyama, A. Yoshikawa, K. Kitazawa, O. Chmaissen, and J. D. Jorgensen, J. Low Temp. Phys. 105, 1379 (1996).

${ }^{13}$ P. Fabbricatore, S. Farinon, G. Gemme, R. Musenich, R. Parodi, and B. Zhang, Phys. Rev. B 50, 3189 (1993).

${ }^{14}$ L. Ji, R. H. Sohn, G. C. Spalding, C. J. Lobb, and M. Tinkham,
Phys. Rev. B 40, 10936 (1989); S. Shatz, A. Shaulov, and Y. Yeshurun, ibid. 48, 13871 (1993); H. Mazaki, H. Yasuoka, M. Kakihana, H. Fujimori, M. Yashima, and M. Yoshimura, Physica C 252, 275 (1995).

${ }^{15}$ N. Morozov, E. Zeldov, D. Majer, and B. Khaykovich, Phys. Rev. Lett. 76, 138 (1996).

${ }^{16}$ L. Burlachkov, A. E. Koshelev, V. M. Vinokur, Phys. Rev. B 54, 6750 (1996).

${ }^{17}$ N. Chikumoto, M. Konczykowski, N. Motohira, and A. P. Malozemoff, Phys. Rev. Lett. 69, 1260 (1992). 